\title{
Tolerancia al calor y humedad atmosférica de diferentes grupos raciales de ganado bovino
}

\section{Tolerance to heat and atmospheric humidity of different breeds groups of cattle}

\author{
José Espinoza V,* Ph.D, Ricardo Ortega P, M.Sc, Alejandro Palacios E, Ph.D, \\ Ariel Guillén T, Ph.D.
}

\begin{abstract}
Universidad Autónoma de Baja California Sur. Departamento de Zootecnia. Cuerpo Académico Producción Animal Sustentable. Carretera al Sur, km. 5.5, La Paz, B.C.S., México. *correspondencia:
\end{abstract} jlvilla@uabcs.mx

Recibido: Junio de 2010; Aceptado: Diciembre de 2010.

\section{RESUMEN}

Objetivo. Comparar algunas respuestas fisiológicas al calor y humedad atmosférica entre bovinos Chinampos (Bos taurus; Ch), 1/2 Charolais 1/2 Brahman (CB), Holstein (H) y Jersey (J). Materiales y métodos. Se realizó un estudio en La Paz, México. Se utilizaron 12 vacas $\mathrm{H}, 10 \mathrm{~J}, 15 \mathrm{Ch}$ y $8 \mathrm{CB}$. Durante el verano se midieron cada semana (a las 0600 y 1600 horas) la frecuencia respiratoria (FR) y temperatura rectal (TR). Se registraron las variables climáticas para calcular el índice de temperatura-humedad (ITH). Resultados. Durante todo el verano, la FR en los cuatro grupos estuvo por encima de los valores fisiológicos para el ganado bovino, siendo superior en $\mathrm{H}$ y J que en $\mathrm{Ch}$ y $C B(p<0.01)$. En todos los grupos, la TR estuvo dentro de los límites fisiológicos de la especie estudiada. El grupo racial más afectado fue $\mathrm{H}(\mathrm{p}<0.05)$. Cuando las constantes fisiológicas fueron analizadas dentro de cada una de tres categorías de ITH ( $<72, \geq 72<78, y \geq 78$ ), las vacas $\mathrm{H}$ y J mostraron FR superior a las Ch y CB $(p<0.01)$. La TR aumentó conforme se elevó el ITH siendo más marcado en J y H. El incremento de la FR entre la mañana y la tarde de cada día de medición fue mayor en $\mathrm{H}$ y J que en $\mathrm{Ch}$ y $\mathrm{CB}(\mathrm{p}<0.05)$. La TR se incrementó más en $\mathrm{H}$ que en $\mathrm{Ch}(\mathrm{p}<0.05)$. Conclusiones. Los grupos raciales más tolerantes al estrés por calor, determinado con base en FR y TR fueron Ch y CB.

Palabras clave: Bovinos, termorregulación, temperatura ambiental. (Fuente: DeCS) 


\section{ABSTRACT}

Objective. The objective of the present study was to compare some physiological responses to heat and atmospheric humidity among Chinampos (Bos Taurus; Ch), 1/2 Charolais $1 / 2$ Brahman (CB), Holstein $(\mathrm{H})$ and Jersey $(\mathrm{J})$ cattle. Materials and methods. The study was carried out in La Paz, Mexico; $12 \mathrm{H}, 10 \mathrm{~J}, 15 \mathrm{Ch}$ and $8 \mathrm{CB}$ cows were used. During the summer the respiratory frequency (RF) and rectal temperature (RT) were measured each week (at 0600 and 1600 hours). The climatic variables were measured to calculate the temperature-humidity index (THI). Results. Throughout the summer, the RF was above the physiological values of cattle in the four groups, being higher in $\mathrm{H}$ and $\mathrm{J}$ than in $\mathrm{Ch}$ and CB $(p<0.01)$. In all groups, the RT was within the physiological limits of cattle. The most affected group was $\mathrm{H}(\mathrm{p}<0.05)$. When the physiological constants were analyzed within each of the three categories of THI $(<72, \geq 72<78$, and $\geq 78)$, the $\mathrm{H}$ and J cows presented higher RF than that of $\mathrm{Ch}$ and $\mathrm{CB}(\mathrm{p}<0.01)$. The RT increased as the THI rose, being more pronounced in $\mathrm{J}$ and $\mathrm{H}$. Increase in the RF between the morning and the afternoon of each day of measurement was higher in $\mathrm{H}$ and $\mathrm{J}$ than in $\mathrm{Ch}$ and $\mathrm{CB}(\mathrm{p}<0.05)$. The RT increased more in $\mathrm{H}$ than in $\mathrm{Ch}(\mathrm{p}<0.05)$. Conclusions. The breed groups most tolerant to heat stress, based on RF and RT, were Ch and CB.

Key words: Bovines, thermoregulation, temperature. (Source: DeCS)

\section{INTRODUCCIÓN}

Entre los factores climáticos que conforman el medio ambiente, la temperatura es uno de los que pueden limitar o condicionar una serie de procesos biológicos, pues el animal se ve obligado a recurrir a determinados mecanismos fisiológicos de regulación térmica en detrimento en su nivel productivo (1). Sin embargo, los efectos del ambiente climático se miden con mayor frecuencia tomando como referencia el índice de temperatura - humedad (ITH) que considera la temperatura ambiente y la humedad relativa (2). Los valores críticos del ITH mínimo, promedio y máximo para el ganado bovino Holstein son de 64, 72 y 76 , respectivamente (3).

Dentro del ganado bovino lechero, una de las razas más susceptibles al estrés calórico es la Holstein (4). El ganado Bos índicus adquirió genes que le confirieron termotolerancia en algún momento durante la separación evolutiva del Bos taurus, haciéndolo más adaptable a los climas calientes (5). Las razas Criollas de Latinoamérica tienen dentro de sus particularidades, una tolerancia elevada a las temperaturas ambientales altas (6).
Estos grupos raciales que evolucionaron en zonas cálidas tienen una mayor capacidad para transferir el calor interno al exterior y regulan con mayor eficiencia su temperatura corporal que el ganado Bos taurus, originario de zonas templadas (7). Recientemente, se encontró que el bovino Chinampo fue menos afectado por la temperatura y la humedad del verano que el ganado Holstein y Jersey cuando se midió la respuesta con base en la temperatura rectal (TR) y la frecuencia respiratoria (FR) (4). El Chinampo de la península de Baja California, en México, tiene su origen en las razas bovinas que fueron introducidas durante la conquista española. Los animales fueron expuestos a un clima caliente y seco durante muchas generaciones y la selección natural produjo un ganado Criollo, conocido localmente como Chinampo (8). Considerando lo anterior, el objetivo del presente trabajo fue estudiar la termotolerancia de vacas Chinampo (Ch), Charolais x Brahman (CB), Hostein ( $\mathrm{H})$ y Jersey $(\mathrm{J})$ tomando como base su temperatura rectal y frecuencia respiratoria. 


\section{MATERIALES Y MÉTODOS}

Sitio de estudio. El trabajo se desarrolló en el municipio de La Paz, localizado al sur de la península de Baja California, México, en las coordenadas geográficas $26^{\circ} 06^{\prime} 01^{\prime \prime}$ $\mathrm{N}$ y $110^{\circ} 00^{\prime} 00^{\prime \prime} \mathrm{O}$, a $33 \mathrm{msnm}$. El clima predominante en la zona (tropical seco), según la clasificación de Köppen se define como BW $(\mathrm{H}) \mathrm{HW}(\mathrm{X})$, siendo este clima seco y cálido con lluvias en verano, invierno y escasas todo el año, con una precipitación pluvial media anual de $195.4 \mathrm{~mm}$ y una temperatura media anual de $28.7^{\circ} \mathrm{C}$ (9).

Diseño del estudio. Se utilizaron 45 vacas adultas $(\mathrm{H}, \mathrm{n}=12 ; \mathrm{J}, \mathrm{n}=10 ; \mathrm{Ch}, \mathrm{n}=15 ; \mathrm{CB}$, $\mathrm{n}=8$ ). Todos los animales se mantuvieron estabulados en corrales contiguos en los que se dispusó sombra y agua. Fueron alimentados con alfalfa henificada y concentrado comercial para cubrir sus requerimientos de energía y proteína de acuerdo con su peso vivo y estado fisiológico (vacas secas, no gestantes).

Entre los meses de mayo hasta septiembre, diariamente se registró la temperatura ambiente máxima mediante un termómetro de bulbo seco, así como la humedad relativa con un psicrómetro (Ertco). En ese lapso (mayoseptiembre), cada lunes se midió en forma individual, la temperatura rectal con un termómetro digital y la frecuencia respiratoria mediante la observación de los movimientos inspiratorios en el abdomen. Esas variables fisiológicas se tomaron dos veces en el mismo día, a las 06:00 y a las 16:00 horas, considerando que son los momentos del día en que los animales tienen la menor y la mayor carga térmica, respectivamente.

Mediante la ecuación de Kibler (10) se calculó el Índice de TemperaturaHumedad (ITH), el cuál expresa la temperatura equivalente a un $100 \%$ de humedad:

$I T H=1.8 \times T a-(1-R H) \times(T a-14.3)+32$

donde $\mathrm{Ta}$ es la temperatura ambiente promedio $\left({ }^{\circ} \mathrm{C}\right)$ y $\mathrm{RH}$ es la humedad relativa promedio expresada en porcentaje.
Considerando que cuando el ITH es superior a 72, las vacas lecheras de alta producción se ven afectadas, el ITH registrado los días de las evaluaciones de las variables de respuesta se clasificó en tres categorías $(<72, \geq 72<78$ y $\geq 78$ ).

Análisis estadístico. Todos los análisis se realizaron usando un modelo lineal general (11). El modelo lineal general (GLM) incluyó el efecto del ITH $(<72, \geq 72<78$ y $\geq 78)$ y el efecto del grupo racial anidado dentro de cada ITH. Las variables dependientes fueron la temperatura rectal y la frecuencia respiratoria. Adicionalmente, de la misma forma se midieron las respuestas fisiológicas de cada raza al cambio en el ITH y las diferencias en las variables dependientes, medidas cada lunes en la mañana y en la tarde. Las medias de mínimos cuadrados fueron comparadas utilizando el procedimiento LSMEANS del SAS (11). La significancia estadística fue aceptada como $\mathrm{p}=0.05$.

\section{RESULTADOS}

Las condiciones climáticas promedio que prevalecieron durante el periodo en que se desarrolló el estudio fueron relativamente estables.

Durante el mes de junio se registró la temperatura máxima más elevada $\left(40.32^{\circ} \mathrm{C}\right)$ y en septiembre la de menor valor $\left(36.22^{\circ} \mathrm{C}\right)$. La humedad relativa promedio más alta se presentó durante septiembre con un $85 \%$ y el mes más seco fue mayo (69.8\%).

El ITH promedio fue de 70.8, 80.1, 83.0, 83.4 y 84.4 en los meses de mayo, junio, julio, agosto y septiembre, respectivamente.

En la tabla 1 se observa que cuando se analizó la FR dentro de cada categoría de ITH, estuvo afectada por el grupo racial. Cuando el ITH fue $<72$, la FR fue menor en el ganado $\mathrm{Ch}$ que en $\mathrm{H}(\mathrm{p}<0.01)$ y similar al resto de los grupos $(P>0.05)$. A este nivel de ITH, el resto de los grupos genéticos tuvieron una FR parecida $(p>0.05)$. Cuando el ITH adquirió valores $\geq 72<78$, la 
Tabla 1. Frecuencia respiratoria (media \pm E.E.) en vacas de diferentes grupos raciales expuestas a distintos niveles de índice de temperatura-humedad (ITH).

\begin{tabular}{|c|c|c|}
\hline \multirow[b]{2}{*}{ Grupo racial } & \multicolumn{2}{|c|}{ Índice de temperatura-humedad ${ }^{1}$} \\
\hline & 1 & 3 \\
\hline Criollo & $37.0 \pm 0.8 \mathrm{a}$ & $37.0 \pm 0.3 a 46.0 \pm 0.3 a$ \\
\hline Charolais x Brahman & $39.8 \pm 1.7 a b$ & $39.7 \pm 0.9 \mathrm{a} 48.2 \pm 0.9 \mathrm{a}$ \\
\hline Holstein & $45.3 \pm 1.7 \mathrm{~b}$ & $49.7 \pm 0.7 b 69.6 \pm 1.0 b$ \\
\hline Jersey & $41.8 \pm 2.3 a b$ & $45.9 \pm 0.9 \mathrm{c} 67.9 \pm 1.4 \mathrm{~b}$ \\
\hline
\end{tabular}

FR del ganado Ch fue más baja que en $\mathrm{H}$ y J $(p<0.01)$ y similar a CB $(p>0.05)$ el cual tuvo una FR más baja que ambas razas lecheras $(p<0.01)$. En este nivel de ITH, las vacas $H$ registraron una $F R$ superior a las J $(p<0.01)$. Cuando el ITH fue $\geq 78$, la FR de las razas para carne (Ch y $C B$ ) fue más baja que en las razas lecheras $(\mathrm{H}$ y J) sin registrarse diferencias dentro de los animales de tipo carne o leche ( $p>0.05)$.

En la figura 1 se observa que, cuando se analizó la respuesta de los animales dentro de cada raza, la FR del ganado $\mathrm{Ch}$ y $\mathrm{CB}$ se mantuvo sin cambio entre los ITH $<72$ $y \geq 72<78(p>0.05)$ y se incrementó de manera significativa $(p<0.01)$ cuando el ITH fue $\geq 78$. La misma tendencia se observó en $\mathrm{H}$ y $\mathrm{J}$, pero en estos animales, aunque la

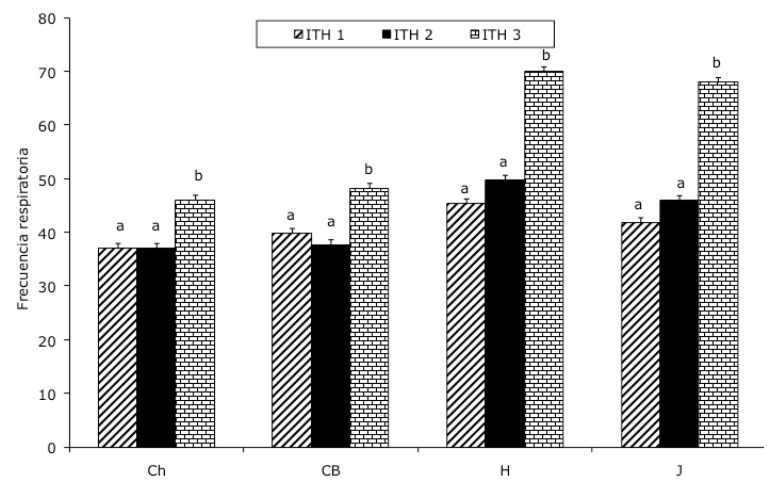

Figura 1. Frecuencia respiratoria (inspiraciones/ minuto) de vacas Chinampas (Ch), $1 / 2$ Charolais $1 / 2$ Brahman (CB), Holstein $(\mathrm{H})$ y Jersey (J) dentro de cada categoría de ITH (ITH1 $=<72$, ITH $2=\geq 72<78$, ITH $3=\geq 78$ ). Literales distintas dentro de cada grupo racial indican desigualdad $(p<0.01)$. elevación de la FR no fue significativa entre los ITH $<72$ y $\geq 72<78$ ( $p>0.05$ ), se observa un cambio importante. El cambio que se registró en la FR de $\mathrm{Ch}$ y $\mathrm{CB}$ entre el ITH más bajo y el más alto, fue numéricamente inferior $(\approx 9$ respiraciones por minuto) que en $\mathrm{H}$ y $\mathrm{J}$ ( $\approx 25$ respiraciones por minuto).

En la tabla 2 se aprecian los valores de la TR en los diferentes grupos raciales dentro de cada categoría de ITH. Cuando Ios ITH fueron $<72$ y $\geq 72<78$, las vacas en los cuatro grupos registraron una TR similar $(p>0.05)$. Cuando el ITH fue $\geq 78$, la TR fue superior en las vacas $\mathrm{H}$ que en Ch, CB y J $(p<0.01)$.

Tabla 2. Temperatura rectal (media \pm E.E.) en vacas de diferentes grupos raciales expuestas a distintos índices de temperatura-humedad.

\begin{tabular}{lccc}
\hline & \multicolumn{3}{c}{ Índice de temperatura-humedad } \\
\cline { 2 - 4 } Grupo racial & 1 & 2 & 3 \\
\hline Criollo & $38.2 \pm 0.1^{\mathrm{a}}$ & $38.6 \pm 0.0^{\mathrm{a}}$ & $39.2 \pm 0.0^{\mathrm{a}}$ \\
Charolais x Brahman & $38.2 \pm 0.2^{\mathrm{a}}$ & $38.5 \pm 0.1^{\mathrm{a}}$ & $39.0 \pm 0.1^{\mathrm{a}}$ \\
Holstein & $37.4 \pm 0.3^{\mathrm{a}}$ & $38.5 \pm 0.0^{\mathrm{a}}$ & $39.7 \pm 0.0^{\mathrm{b}}$ \\
Jersey & $37.5 \pm 0.2^{\mathrm{a}}$ & $38.4 \pm 0.0^{\mathrm{a}}$ & $38.9 \pm 0.0^{\mathrm{a}}$ \\
\hline
\end{tabular}

$\mathrm{a}, \mathrm{b}, \mathrm{c}$ Literales diferentes en las columnas indican desigualdad $(p<0.01) .{ }^{1} \mathrm{ITH} 1=<72, \mathrm{ITH} 2=\geq 72<78, \mathrm{ITH} 3=\geq 78$.

Como se expresa en la figura 2, los cuatro grupos raciales registraron un incremento significativo de su TR conforme el ITH

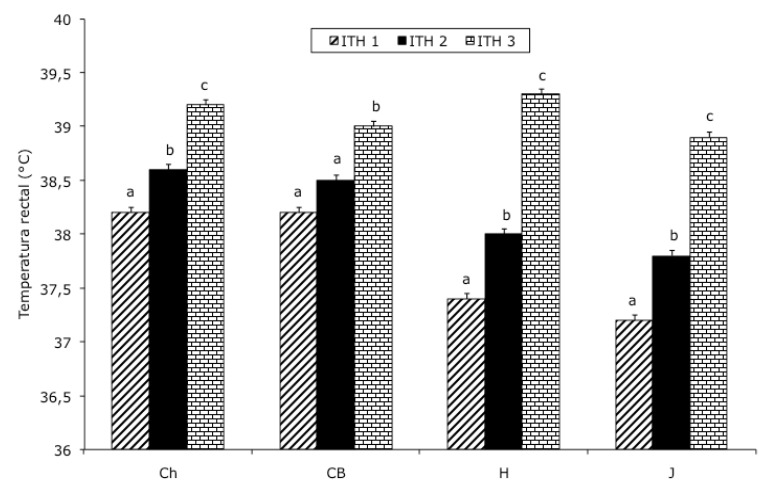

Figura 2. Temperatura rectal $\left({ }^{\circ} \mathrm{C}\right)$ de vacas Chinampas (Ch), $1 / 2$ Charolais $1 / 2$ Brahman (CB), Holstein (H) y Jersey (J) dentro de cada categoría de ITH $\quad$ (TTH $1=<72, \quad$ ITH $2=\geq 72<78$, ITH3 $=\geq 78$ ). Literales distintas dentro de cada grupo racial indican desigualdad $(p<0.01)$. 
aumentó de $<72$ a $>78 \quad(p<0.01)$. Se puede ver que el cambio de la TR entre los dos extremos de ITH fue más intenso en las razas lecheras $\left(1.9\right.$ y $1.7^{\circ} \mathrm{C}$ en $\mathrm{H}$ y $\mathrm{J}$, respectivamente) que en $\mathrm{Ch}\left(1^{\circ} \mathrm{C}\right)$ y $\mathrm{CB}\left(0.8^{\circ} \mathrm{C}\right)$.

En la tabla 3 se presentan las diferencias observadas en las FR y las TR registradas por la mañana y por la tarde de cada día de medición. Las vacas más afectadas por la

Tabla 3. Diferencia (Media $\pm E E$ ) entre las mediciones realizadas por la mañana y en la tarde de cada día de evaluación de la frecuencia respiratoria (FR) y la temperatura rectal (TR).

\begin{tabular}{|c|c|}
\hline & Constante fisiológica \\
\hline Grupo racial & $\begin{array}{cc}\mathrm{FR} & \mathrm{TR}\left({ }^{\circ} \mathrm{C}\right) \\
\end{array}$ \\
\hline Chinampo & $10.0 \pm 0.3 a \quad 0.8 \pm 0.0 a$ \\
\hline $\begin{array}{l}1 / 2 \text { Charolais } 1 / 2 \\
\text { Brahman }\end{array}$ & $13.1 \pm 0.8 \mathrm{a} 1.0 \pm 0.1 \mathrm{ab}$ \\
\hline Holstein & $23.2 \pm 1.5 b \quad 1.4 \pm 0.1 b$ \\
\hline Jersey & $25.2 \pm 2.2 \mathrm{~b} \quad 1.0 \pm 0.1 \mathrm{ab}$ \\
\hline
\end{tabular}

a,bLiterales diferentes en las columnas indican desigualdad $(p<0.05)$.

elevación de los valores climáticos durante el día fueron $\mathrm{H}$ y J. La FR se incrementó más en estas dos razas que en $\mathrm{Ch}$ y $\mathrm{CB}$ $(p<0.05)$. De manera similar, la TR se incrementó más en las vacas $\mathrm{H}$ que en las Ch $(p<0.05)$.

\section{DISCUSIÓN}

Los valores de temperatura máxima que se presentaron en este estudio superan en todo momento a la temperatura crítica alta en vacas lecheras, la cual varía entre 25 y $26^{\circ} \mathrm{C}(12)$.

Los valores de ITH registrados son superiores a los encontrados por otros en la misma zona (13), debido probablemente a que, durante el periodo en el que se desarrolló el presente trabajo, la humedad relativa fue más elevada. El ITH promedio estimado en este trabajo desde mayo hasta septiembre se encuentra por encima del nivel de confort crítico $(\leq 72)$ para el ganado Holstein (14).
Todas las razas se vieron afectadas por el calor y la humedad ya que la FR se mantuvo por encima de los valores normales de 24 a 36 ciclos respiratorios por minuto (15) desde mayo hasta septiembre. Sin embargo, se puede apreciar que las razas lecheras $(\mathrm{H}$ y $\mathrm{J})$ incrementaron más su FR que las vacas $C h$, sin registrarse diferencias entre $\mathrm{H}$ y J. Esto coincide con el aumento registrado en el ITH, el cual pudo haber reducido la sudoración y como un recurso termorregulatorio adicional, pudo haberse incrementado la evaporación a través de la vía respiratoria (16). En ambientes cálidos, la respiración es lo primero que reacciona en el bovino como mecanismo adicional de ventilación pulmonar y evaporación para mantener la hemotermia, si este gasto fisiológico adicional fracasa, se eleva la TR (4). El ganado incrementa su FR, ventilación pulmonar y vaporización respiratoria cuando aumenta la temperatura ambiente, pero cuando las temperaturas alcanzan los $26.7^{\circ} \mathrm{C}$ en el $B$. taurus y $35^{\circ} \mathrm{C}$ en el $B$. índicus, esos mecanismos son incapaces de disipar todo el exceso de calor (17).

La FR más baja y consistente que se observó en Ch y CB durante este estudio se puede interpretar como una mejor adaptación al calor que las razas lecheras, ya que regularmente es a través de una menor FR durante periodos calurosos como se identifican los animales menos afectados por las altas temperaturas (18) y podría ser un indicativo de una mayor área superficial por masa corporal para disipar el calor o una menor tasa metabólica que los otros genotipos (19).

Además, es importante considerar que el ganado $B$. índicus, adquirió genes que le confirieron termotolerancia en algún momento durante la separación evolutiva del $B$. taurus haciéndolo más adaptable a los climas calientes (5) y este podría ser el caso de las vacas CB del presente trabajo. Una FR similar a la registrada en los animales de este estudio fue reportada en diferentes razas de ganado Criollo de Brasil (20). Valores más altos en la FR fueron constatadas en ganado de pelo corto (56 inspiraciones/minuto) y en ganado con pelo normal (69 inspiraciones/minuto), ambos 
grupos producto de la cruza de Holstein con Senepol (18).

Los valores correspondientes a los novillos Holstein de ese estudio son superiores a los observados en el presente trabajo para la misma raza y las FR que se registraron en el ganado Brahman y en las cruzas de Holstein con las razas termotolerantes son similares a las que se obtuvieron en el ganado Ch de esta investigación.

En vacas Holstein en Venezuela, se registró una FR de 73.8 cuando el ITH fue de 87 (1). En otro trabajo (21), cuando el ITH fue de 85 , la FR fue de 69 y 64 en vaquillas Angus y Hereford, respectivamente y se mantuvo dentro de los límites normales en los animales Brahman, no obstante en otras razas de ganado termotolerante como el Senepol y Romosinuano se registraron 55 y 57 respiraciones por minuto. Los valores obtenidos en las razas resistentes al estrés calórico en ese estudio resultan superiores a la FR observada en el ganado Ch de esta investigación.

En las tres categorías del ITH, la TR se mantuvo dentro de los límites normales de 37.5 a $39.5^{\circ} \mathrm{C}(22)$.

Las diferencias entre grupos raciales observadas en la TR no son consistentes con la variación obtenida en la FR. Sin embargo, bajo condiciones de estrés calórico la FR empieza a elevarse antes que la TR (23) y generalmente se observa taquipnea en bovinos expuestos a ambientes con temperatura elevada (24).

Los valores de TR observados en el presente estudio resultan más altos que los reportados en vacas Holstein expuestas a ITH que fluctuaron entre 68 y 78 (25). Son parecidos a los obtenidos en Brasil en la misma raza cuando los animales se mantuvieron bajo un ITH de 80 (26).
En un estudio realizado con bovinos cruzados (B. taurus $\times$ B. índicus) durante los meses de julio y agosto, se observaron TR similares al presente estudio cuando los ITH fueron de 81.5 y 80.1 , respectivamente (22).

En Florida, cuando el ITH fue de 85, la TR superó los $40^{\circ} \mathrm{C}$ en vaquillas Angus y Hereford y se mantuvo dentro de los límites normales $\left(\leq 39.5^{\circ} \mathrm{C}\right)$ en los animales Brahman, Senepol y Romosinuano (21). Resultados similares a los observados en el Romosinuano y Senepol del trabajo anterior fueron constatados en el presente estudio.

En un estudio reciente se comparó la respuesta al estrés calórico entre novillos Angus y Romosinuanos mantenidos en ambiente controlado y se observó que en ambas razas aumentaron la FR y la TR al someterlas a estrés por calor (27).

En conclusión, de acuerdo con los resultados obtenidos en este trabajo, se concluye que las condiciones climáticas fueron relativamente constantes entre junio y septiembre, con ligeras variantes que se vieron reflejadas en las respuestas fisiológicas de los animales entre los meses mencionados. Los grupos raciales más termotolerantes, determinados con base en la respuesta de su frecuencia respiratoria y temperatura rectal, fueron el Chinampo y la cruza $1 / 2$ Charolais $1 / 2$ Brahman. Dentro de las dos razas lecheras, el ganado Holstein se vio más afectado por el índice de temperatura-humedad. La mayor tolerancia de las vacas Chinampas y las $1 / 2$ Charolais $1 / 2$ Brahman se vio reflejada también en un menor aumento de su frecuencia respiratoria en las horas críticas del día, con respecto a las razas lecheras. 


\section{REFERENCIAS}

1. Valle A. Importancia del porcentaje de área negra en animales Holstein sobre el proceso adaptativo. III. Respuestas fisiológicas a la exposición solar directa. Zoot Trop 1985;2:3-20.

2. NOAA. Livestock hot weather stress. United States Dept of Commerce, Natl Oceanic and Atmospheric Admin, Natl Weather Service Central Region. USA: Regional Operations Manual Letter; 1976

3. Igono MO, Bjotvedt G, Sanford-Crane HT. Environmental profile and critical temperature effects on milk production of Holstein cows in desert climate. Int J Biometeorol 1992;36:77-87.

4. Espinoza JL, Sánchez J, Gracia JA, Sánchez JR, Ortega R, Palacios A. Thermoregulation differs in Chinampo (Bos Taurus) and locally born dairy cattle. Turk J Vet Anim Sci 2009;33:175-180.

5. Hansen PJ. Physiological and cellular adaptations of zebu cattle to thermal stress. Anim Reprod Sci 2004;8283:349-360.

6. Martínez BA. La ganadería en Baja California Sur, La Paz BCS México: Ed JB; 1981.

7. Finch VA. Body temperature in beef cattle: its control and relevance to production in the tropics. J Anim Sci 1986; 62:531-542.

8. Espinoza JL, Guevara A, Palacios A. Caracterización morfométrica y faneróptica del bovino Criollo Chinampo de México. Arch Zootec 2009; 58:277-279.

9. DGTENAL. Carta topográfica. Dirección General de Geografía del Territorio Nacional, SPP; La Paz, Baja California Sur, México: G12D83; 1980.
10. Ravagnolo O, Misztal I, Hoogenboom G. Genetic component of heat stress in dairy cattle, development of heat index function. J Dairy Sci 2000; 83:21202125.

11. SAS. SAS/STAT User's Guide (Release 6, 4th Ed.). Cary NC: SAS. Inst. Inc; 1990.

12. Berman A, Folman YM, Kaim M, Mamen Z, Herz D, Wolfenson A, Graber Y. Upper critical temperatures and forced ventilation effects for high-yielding dairy cows in a tropical climate. J Dairy Sci 1985; 68:488-495.

13. Sánchez-Ríos J. Influencia de factores climáticos sobre algunas constantes fisiológicas en vacas de diferentes grupos raciales. [Tesis de Licenciatura]. La Paz, México: Universidad Autónoma de Baja California Sur, Departamento de Zootecnia; 2007.

14. Armstrong DV. Heat stress interaction with shade and cooling. J Dairy Sci $1994 ; 77: 2044-2050$.

15. Marai IFM, Habeeb AAM, Farghaly HM. Productive, physiological and biochemical changes in imported a locally born Holstein lactating cows under hot summer conditions of Egypt. Trop Anim Health Prod 1999; 31:233-243.

16. Coppock CE, Grant PA, Portzer SJ, Charles DA Escobosa A. Lactating dairy cow responses to dietary sodium, chloride, and bicarbonate during hot weather. J Dairy Sci 1982; 65:566576.

17. Brody S. Climatic physiology of cattle. J Dairy Sci 1956; 39:715-725.

18. Ávila M. Physiological responses of slick versus normal haired Holstein $\mathrm{X}$ Senepol crossbred cattle in Florida. [MS Thesis] USA: Universidad Autónoma de Baja California Sur, Department of Animal Sci; 2002. 
19. Gaughan JB, Mader TL, Holt SM, Josey MJ Rowan KJ. Heat tolerance of Boran and Tuli crossbred steers. J Anim Sci 1999; 77:2398-2405.

20. McManus $C$, Paludo GR, Louvandini $H$, Garcia JAS, Egito AA, Mariante AS. Heat tolerance in naturalized cattle in Brazil: physical factors. Arch Zootec 2005: 54:453-458.

21. Hammond AC, Olson TA, Chase Jr CC, Bowers EJ, Randel RD, Murphy CN, et al. Heat tolerance in two tropically adapted Bos taurus breeds, Senepol and Romosinuano, compared with Brahman, Angus, and Hereford cattle in Florida. J Anim Sci 1996; 74:295-303.

22. Alzina-López A, Farfán-Escalante JC, Valencia-Heredia ER, Yokohama-Kano J. Condición ambiental y su efecto en la temperatura rectal y frecuencia respiratoria en bovinos cruzados (Bos taurus x Bos índicus) del estado de Yucatán, México. Rev Biomed 2001; 12:112-121.

23. Bianca W. Review of the progress of dairy science: Cattle in hot environment. J Dairy Sci 1965; 32:291-308.
24. Ferreira $F$, Pires MFA, Martinez ML, Coelho SG, Carvalho AU, Ferreira PM, et al. Parâmetros fisiológicos de bovinos cruzados submetidos ao estresse calórico. Arq Bras Med Vet Zootec 2006; 58:732-738.

25. Bouraoui R, Lahmar M, Majdoub A, Djemali $M$, Belyea $R$. The relationship of temperature-humidity index with milk production of dairy cows in a mediterranean climate. Anim Res 2002; 51:479-491.

26. Barbosa OR, Boza $P$, dos Santos G, Sakagushi E, Ribas N. Efeitos da sombra e da aspersão de água na produção de leite de vacas da raça Holandesa durante o verão. Acta Scientiarum Anim Sci Maringá 2004; 26:115-122.

27. Scharf B, Carroll JA, Riley DG, Chase Jr CC, Coleman SW, Weaber RL, et al. Evaluation of physiological and blood serum differences in heat-tolerant (Romosinuano) and heat-susceptible (Angus) Bos taurus cattle during controlled heat Challenger. J Anim Sci 2010;88:2321-2336. 